\title{
- Case Report . \\ Malignant ovarian melanoma with extensive pelvic and peritoneal metastasis: a case report and literature review
}

\author{
Rong Gao, Nai-Fu Liu, Xiu-Gui Sheng \\ Department of Gynecologic Oncology, Shandong Tumor Hospital and Institute, Jinan, Shandong 250117, P.R. China
}

Key words: Ovarian teratoma, malignant melanoma, pelvic and peritoneal metastasis

Primary malignant ovarian melanoma, which is primarily transformed from ovarian mature teratoma, is rare ${ }^{[1,2]}$. Each component of ovarian mature teratoma can be transformed to malignant tumor, with the squamous carcinoma as the most common type accounting for about $80 \%$, followed by adenocarcinoma, and those transformed to melanoma are rare ${ }^{[3]}$. We reported a case of teratoma-transformed malignant ovarian melanoma with extensive pelvic and peritoneal metastasis. This patient had teratoma involving bilateral ovaries, with one side transformed to malignant ovarian melanoma. By reviewing the literature, we discussed the pathogenesis, clinicopathologic features, differential diagnosis and treatment of teratoma-transformed malignant ovarian melanoma.

\section{Case presentation}

A 53-year old woman was admitted to Shandong Tumor Hospital on June 2, 2008 because of abdominal distension for half a month. Physical examination revealed that there was no melanotic nevus or pigmentation on the skin or mucus, abdominal shift dullness was positive, a mass measured $8 \mathrm{~cm} \times$ $7 \mathrm{~cm}$ was palpated at the left posterior side of the uterus, and nodules were palpated in the rectouterine pouch. CT scan revealed a pelvic mass, considered to be ovarian cancer with ascites formation. The serum level of CA125 was $351.7 \times 10^{3}$ $\mathrm{U} / \mathrm{L}$. Paracentesis and tube drainage were performed, obtaining a total volume of $6900 \mathrm{~mL}$ dark-brown ascites. Cytology of the asictes revealed malignant cells (malignant melanoma). The gastroscopy and colonoscopy showed chronic gastritis and ulcerative colitis. The patient was treated by intraperitoneal lavage with $60 \mathrm{mg}$ cisplatin plus $2000000 \mathrm{U}$ interleukin-2, one cycle of systemic chemotherapy with paclitaxel plus cisplatin, and

Correspondence to: Rong Gao; Tel: +86-531-67626546; Fax: +86-531-85858077; Email: gaorong_sd@163.com

This paper was translated from Chinese into English by CJC Medical Translation and edited by Wei Liu on 2010-03-09.

The Chinese version of this paper is avaiable at http://www.cjcsysu.cn/cn/article .asp?id=16403.

Received: 2009-08-31; Accepted: 2010-02-08 surgical exploration. The operation revealed $800 \mathrm{~mL}$ light-yellow ascites in the abdominal cavity; the pelvic peritoneum, posterior peritoneum and posterior wall of the bladder were all covered with melanoma tissues, and intestinal wall and mesentery were black brown in color (Figure 1); several black tumor nodules measured about $2.5 \mathrm{~cm} \times 3 \mathrm{~cm} \times 3 \mathrm{~cm}$ were palpated in the abdominal wall and peritoneum; the uterus was atrophic with a large amount of black spots scattered on the serosa of uterus fundus; the left ovary was measured $8 \mathrm{~cm} \times 6 \mathrm{~cm} \times 5 \mathrm{~cm}$ in size with irregular appearance and ruptured capsule (Figure 2); the right ovary was measured $4 \mathrm{~cm} \times 3 \mathrm{~cm} \times 3 \mathrm{~cm}$ in size with intact capsule; both ovaries presented alternate colors of white and black; both fallopian tubes appeared normal; a black and firm nodule measured $4 \mathrm{~cm} \times 2 \mathrm{~cm} \times 2 \mathrm{~cm}$ was seen in the rectouterine pouch.

Tumor cells were extensively seeded in the pelvic and abdominal cavities; the lesions were diffuse and impossible to be radically dissected. We performed resection of bilateral adnexa. Postoperative pathology showed a large amount of melanoma cells arranged in patchy pattern in the left adnexa; the atypia of these cells was remarkable with enlarged nuclei and many cells had red nucleoli; nuclear mitosis was easily identifiable(Figure 3), and there was components of squamous or ciliated columnar epithelia; the right adnexa showed mature cystic teratoma with malignant melanoma tissues on the surface. Immunohistochemical assay showed that HMB45, Vimentin and S-100 proteins were extensively expressed. The pathologic diagnosis was malignant ovarian melanoma derived from teratoma (left ovary), mature cystic teratoma with tumor cells on the surface (right ovary). After operation, $3000000 \mathrm{u}$ of interferon was injected subcutaneously every other day, totally 10 times; one cycle of systemic intravenous chemotherapy with 40 $\mathrm{mg}$ cisplatin, $400 \mathrm{mg}$ dacarbazine plus $16 \mathrm{mg}$ bleomycin given on days 1-3. The patient lost to follow-up after half a year.

\section{Discussion}

\section{Histological origin}

Malignant melanoma accounts for $3 \%$ of the malignancy of female reproductive system, which is commonly seen in the vulva, vagina and cervix and less commonly seen in the uterus 


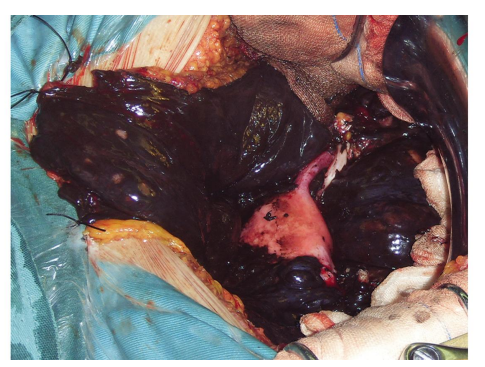

Figure 1 Diffuse peritoneal metastatic melanoma The pelvic peritoneum and the posterior wall of the bladder are extensively covered by melanoma tumor tissues, the intestine and mesentery are dark brown, and the uterine serosal shows scattered black spots.

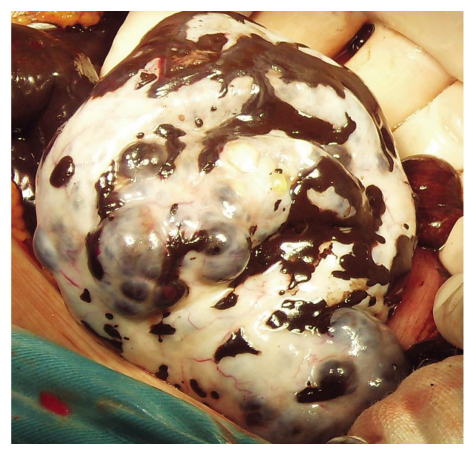

Figure 2 The left ovarian tumor

The left ovary is about $8 \mathrm{~cm} \times 6 \mathrm{~cm} \times 5 \mathrm{~cm}$, the capsule is ruptured, and the ovarian surface is covered with black and white spots.

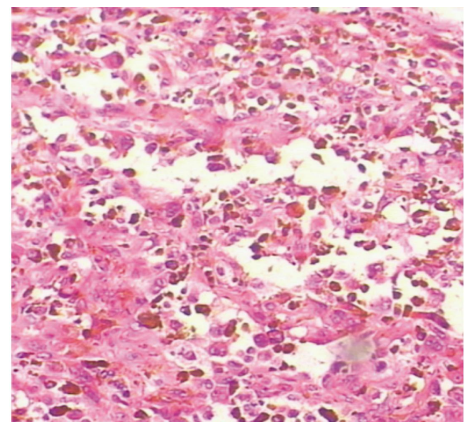

Figure 3 Ovarian melanoma cells

A large number of melanoma tumor cells are arranged as sheets, with obvious atypia and enlarged nuclei. Red nucleoli and mitosis are commonly seen.

body and ovary ${ }^{[4,5]}$. Normal ovarian tissue contains no melanoblast. Nonetheless, the skin, retina, meninges, naive neural crest, and so on, in ovarian teratoma contain melanoblasts. The malignant transformation of melanoblasts can result in malignant melanoma. Teratoma-transformed malignant ovarian melanoma is rare, accounting for about $2 \%$ of malignant ovarian melanomas of the female reproductive system, and $0.1 \%$ of ovarian teratoma ${ }^{[6]}$. From the first case of teratoma-transformed malignant ovarian melanoma reported by Amann in 1903 to the year of 2005 , only 32 cases of malignant ovarian melanoma were reported ${ }^{[7]}$. In 2006, McCluggage et al. ${ }^{[8]}$ reported nine cases of primary malignant ovarian melanoma, including six cases with coexisting mature cystic ovarian teratoma.

\section{Differential diagnosis}

Primary malignant ovarian melanoma should be differentiated from metastatic melanoma in the ovary. The former usually involves unilateral ovary, and bilateral involvement is less common; the latter has primary lesion and $95 \%$ involves bilateral ovaries ${ }^{[9]}$. Cronje et al. ${ }^{[10]}$ proposed the diagnostic criteria of primary malignant ovarian melanoma in 1981: (1) no extra-ovarian primary tumor exists; (2) unilateral ovarian tumor with component of teratoma; (3) there are evidences of melanocytes; (4) symptoms and age of the patient are consistent with those described in literature. Zhang et al. ${ }^{[11]}$ reported one case of bilateral primary malignant ovarian melanoma in 1984. In that case, squamous cells derived from ectoderm and metaplastic squamous epithelia derived from endoderm, as components of mature teratoma, as well as a large amount of melanocytes were observed in the left ovary; the right ovary showed benign teratoma, with a large amount of metastatic melanocytes on the surface of the right ovary, pelvic and abdominal cavity. These were consistent with diagnostic criteria.

In addition to metastatic ovarian melanoma, peritoneal melanosis should also be ruled out. Peritoneal melanosis is rare, which manifests as pigmentation of the peritoneum, mesentery, appendix surface, pelvic peritoneum and the surface of ovary. It is divided into primary and secondary forms. The secondary peritoneal melanosis is derived from ovarian teratoma or developmental malformation of gastrointestinal tract. Secondary peritoneal melanosis of ovarian tumor was firstly described by Afonsoy et al. ${ }^{[2]}$ in 1962 . Seven cases were reported in other countries, including 6 with coexisting mature cystic teratoma and 1 with serous ovarian cystadenoma[13-18]. Four cases were reported in China, including 3 with coexisting mature cystic teratoma and 1 with serous cystadenoma ${ }^{[19,20]}$. The clinical manifestations and abdominal exploration findings of peritoneal melanosis mimic primary malignant ovarian melanoma, but the former has benign histological structures and cellular morphology, with inactive nuclear mitosis, lacking features of infiltration and metastasis. The prognosis of such patients is desirable.

\section{Diagnosis and treatment strategy}

Malignant ovarian melanoma is difficult to be confirmed and is most likely to be diagnosed as ovarian cancer preoperatively. If preoperative paracentesis shows black or black-brown ascites, malignant melanoma should be considered. If cytology revealed malignant melonocytes, malignant melanoma is highly indicated. Malignant melanoma is malignant that is prone to distant metastasis with poor prognosis, and patients usually die within 2 years after diagnosis. Chemotherapy can only release symptoms without changing the outcomes. Ovarian tumor cell reduction combined biological chemotherapy is a desirable modality. Many investigators found that the effectiveness rate of the combination of chemotherapy and cytokines (biological therapy) could be improved to as high as $60 \%-70 \%$, and the survival time can be prolonged and quality of life can be improved ${ }^{[21]}$. The efficacy is 


\section{Chinese Journal of Cancer}

obviously better than that of single chemotherapy or biological therapy. The most commonly used chemotherapeutic drugs are dacarbazine, carmustine, cisplatin, vincristine and temozolomide. The commonly used agents for biological therapy are interferon and interleukin-2.

\section{References}

[1] Fan LD. Clinical and pathologic features of the ovary [M]. Tianjin: Tian Xiong Science Press, 1993:283. [in Chinese]

[2] Yan XJ. Ovarian tumor [M]. Beijing: Science and Technology Press, 2009: 100. [in Chinese]

[3] Lian LJ. Lin Qiao-Zhi Gynecologic Oncology [M]. Beijing: People's Medical Publishing House, 2006: 604-605. [in Chinese]

[4] Balch CM, Soong S. Long-term results of a multi-institutional randomized trial comparing prognostic factors and surgical results for intermediate thickness melanomas (1.0 to $4.0 \mathrm{~mm}$ ). Intergroup melanoma surgical trial [J].Ann Srug Oncol, 2000, 7(2):87-97.

[5] Wang YJ, Chang WQ, Pu DW. Clinical Analysis of 15 Patients with Primary Malignant Melanoma in the Genital Tract [J]. Obstet Gynecol, 1997,32(2):226-228. [in Chinese ]

[6] Wang ZM. A Retrospective Study of 887 Cases of Ovarian Teratoma [J]. Tian Jin Yi Yao, 1980, 3(3): 136. [in Chinese ]

[7] McNeilage LJ, Morgan J, Constable J, et al. Metastatic malignant melanoma arising in a mature ovarian cystic teratoma: a case report and literature review [J]. Gynecol Cancer, 2005, 15(6):1148-1152.

[8] McCluggage WG, Bissonnette JP, Young RH. Primary malignant melanoma of the ovary: a report of 9 definite or probable cases with emphasis on their morphologic diversity and mimicry of other primary and secondary ovarian neoplasms [J]. Gynecol Pathol, 2006, 25(4):321329 .

[9] Carlson J A, WheelerJE. Primary ovarian melanoma arising in a dermoid stage IIIC: long-term disease-free survival with aggressive surgery and platinum therapy [J].Gynecol Oncol,1993,48(3):397-401.

[10] Cronje HS, Woodruff JD. Primary ovarian malignant melanoma arising in cystic-teratoma [J]. Gynecol Oncol, 1981, 12(3):379-383.

[11] Zhang PN, Zhu LH. Primary ovarian malignant melanoma with Meigs syndrome: a case report [J]. Zhe Jiang Yi Xue, 1984, 6:46. [in Chinese ]

[12] Afonsoy JF, Martin G, Nisco FS.Melanogenic ovarian tumors [J].Am J Obstet Gynecol, 1962, 84:667-676.

[13] Lee D, Pontifex AH. Melanosis peritoneal [J].Am J Obstet Gynecol, 1975, 122(4):526-527.

[14] Fukushima M, Sharpe L, Okagaki T. Peritoneal melanosis secondary to a benign dermoid cyst of the ovary: a case report with ultrastrctural study [J].Int J Gynecol Pathol, 1984, 2(4):403-409.

[15] Sahin AA, Ro JY, Chen J, et al. Spindle cell nodule and peptic ulcer arising in a fully developed gastric wall in a mature cystic teratoma [J]. Arch Pathol Lab Med, 1990,114(5):529-531.

[16] Kagiya A, Fukushi A, Tachizaki T, et al. A case of melanosis ovarii at peritonei [J]. Nippon Sanka Gakkal Zasshi, 1990,42(1):1571-1574.

[17] Jaworski RC, Boadle R, Greg J, et al. Peritoneal melanosis associated with a ruptured ovarian dermoid cyst: report of a case with electronprobe energy dispersive X-ray analysis [J]. Int J Gynecol Pathol, 2001, 20(4):386-389.

[18] Kin NR, Suh YL, Song SY, et al. Peritoneal melanosis combined with serous cystadenoma of the ovary: a case report and literature review [J] Pathol Int, 2002, 52(11):724-729.

[19] Zhang XS, Zhang LL, Cui QX, et al. Peritoneal melanosis combined with ovarian tumors: a clinicaopathologic study [J]. Clin Exp Pathol, 2007,23 (2):167-170. [in Chinese]

[20] Sun HG, Shen XE, He YM. Peritoneal melanosis combined with gliomatosis: a case report [J]. Chin J Diagn Pathol, 2006,13(3):224-225 [in Chinese ]

[21] Khayat D, Bernard-Marty C, Meric JB, et al. Biochemotherapy for advanced melanoma: maybe it is real [J].Am Clin Oncol, 2002, 20(10): 2411-2414. 